\title{
Ergodic Isoenergetic Molecular Dynamics for Microcanonical-Ensemble Averages
}

\author{
Wm.G. Hoover, C.G. Hoover \\ Ruby Valley Research Institute \\ Highway Contract 60, Box 601, Ruby Valley, Nevada 89833, USA \\ E-mail: hooverwilliam@yahoo.com
}

Received: 25 June 2018; revised: 26 June 2018; accepted: 26 June 2018; published online: 30 June 2018

\begin{abstract}
Considerable research has led to ergodic isothermal dynamics which can replicate Gibbs' canonical distribution for simple (small) dynamical problems. Adding one or two thermostat forces to the Hamiltonian motion equations can give an ergodic isothermal dynamics to a harmonic oscillator, to a quartic oscillator, and even to the "Mexican-Hat" (doublewell) potential problem. We consider here a time-reversible dynamical approach to Gibbs' "microcanonical" (isoenergetic) distribution for simple systems. To enable isoenergetic ergodicity we add occasional random rotations to the velocities. This idea conserves energy exactly and can be made to cover the entire energy shell with an ergodic dynamics. We entirely avoid the Poincaré-section holes and island chains typical of Hamiltonian chaos. We illustrate this idea for the simplest possible two-dimensional example, a single particle moving in a periodic square-lattice array of scatterers, the "cell model".
\end{abstract}

Key words: chaos, ergodicity, Lyapunov exponent, algorithms

\section{INTRODUCTION}

In 1984 Shuichi Nosé discovered a dynamics consistent with Gibbs' canonical ensemble [1,2] at a fixed temperature $T$. Nosé's idea, applied to $N$ unit-mass particles with coordinates and momenta $\{q ; p\}$ is most simply implemented with the Nosé-Hoover motion equations [3]:

$$
\begin{aligned}
& \{\dot{q}=p ; \dot{p}=F-\zeta p\} ; \\
& \dot{\zeta}=\sum^{N}\left[\left(p^{2} / T\right)-1\right] / N \tau^{2}
\end{aligned}
$$

[Thermostatted Dynamics].

The additional single "thermostat variable" $\zeta$ is a "friction coefficient". When negative it injects kinetic energy into the $(q, p)$ system. When positive, kinetic energy is extracted. This integral-feedback form of the motion equations has been selected to be exactly consistent with Gibbs' canonical distribution, $f(q, p) \propto e^{-\mathcal{H} / k T}$, as one of us pointed out in 1985 [3]. The independent variable in the canonical ensemble is the mean value of the kinetic energy, $K(p)=\sum\left(p^{2} / 2 m\right) \equiv$ $\sum\left(p^{2} / 2\right)$. In $D$ spatial dimensions the kinetic energy corre- sponds to Gibbs' (kinetic) temperature, $2 K=D k T \equiv D T$. Throughout our work we set the particle mass $m$ and Boltzmann's constant $k$ both equal to unity, for simplicity.

Nosé's original work used Hamiltonian mechanics. It soon became evident that his thermostatted motion equations were not "ergodic". That is, the $(q, p)$ phase-space states generated by his equations of motion failed to sample the entire phase space and instead sampled only a subspace determined by the initial conditions. Just as in conventional Hamiltonian mechanics two kinds of solutions of Nosé's equations of motion were found, regular solutions, corresponding to simple tori in the phase space, and chaotic Lyapunov-unstable solutions, forming a fat-fractal "chaotic sea". Several years later two-thermostat motion equations were developed. Applied to the harmonic oscillator $[4,5]$ their solutions matched Gibbs' canonical distribution with an ergodic "chaotic sea" and without any regular toroidal solutions.

Much later [6,7] ergodic solutions with only a single thermostat variable were discovered. One was found as the result of a two-parameter computerized search [6]:

$$
\left\{\dot{q}=p ; \dot{p}=-q-0.05 \zeta p-0.32 \zeta\left(p^{3} / T\right)\right\} ;
$$



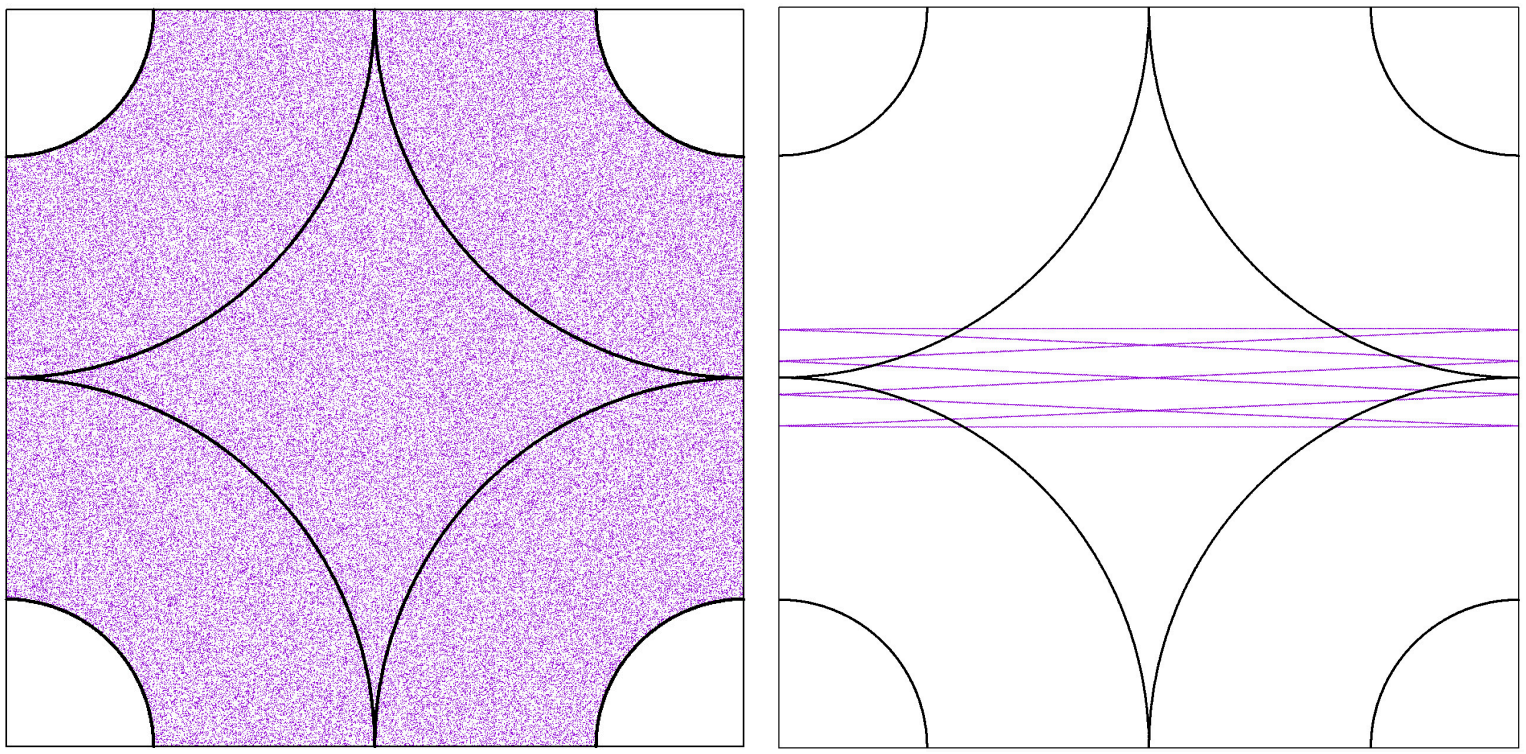

Fig. 1. Cell-Model dynamics with four fixed soft-disk scatterers at the corners of a $2 \times 2$ periodic cell. For the initial condition $q=(0,0) ; p=(0.6,0.8) 200,000$ projections from 200,000,000 timesteps, fourth-order Runge-Kutta, equally spaced in time with $\Delta t=1000 d t=1$, are shown at the left. At the right the initial momenta are $\left(0.999, \sqrt{1-0.999^{2}}\right)$. Evidently this Newtonian problem is not ergodic. The black quadrant lines border the accessible regions where the forces are nonzero. In the central diamond-shaped region the scatterer forces all vanish

$\dot{\zeta}=0.05\left[\left(p^{2} / T\right)-1\right]+0.32\left[\left(p^{4} / T^{2}\right)-3\left(p^{2} / T\right)\right]$

[0532 Model].

A more general one-parameter thermostat, able to generate ergodic solutions to the quartic-well and Mexican-Hat doublewell problems as well as the oscillator, was developed by Tapias, Bravetti, and Sanders as their solution of the 2016 Snook Prize problem [7]:

$$
\ddot{q}=-q-2 \alpha \dot{q} \tanh (\alpha \zeta) ; \dot{\zeta}=\dot{q}^{2}-1 .
$$

There are several tests that a set of motion equations must pass to establish its ergodicity. Any ergodic canonical dynamics algorithm must follow Gibbs' measure in phase space, $f_{\text {Gibbs }}(q, p)=e^{-\mathcal{H} / T} / \int d q \int d p e^{-\mathcal{H} / T}$. Such an algorithm must also generate the canonical averages of the various moments of the potential and kinetic energies. For the canonical oscillator problem the first few nonvanishing moments are the following:

$$
\begin{aligned}
& \left\langle q^{2}, p^{2} ; q^{4}, q^{2} p^{2}, p^{4} ; q^{6} \ldots\right\rangle= \\
= & T, T ; 3 T^{2}, T^{2}, 3 T^{2} ; 15 T^{3} \ldots
\end{aligned}
$$

The Kolmogorov-Arnold-Moser Theorem indicates that molecular dynamics is seldom ergodic, though the consequences of that lack are thought to be small in most cases. A clear lack of ergodicity is shown in Fig. 1, where a single mass point moves in a periodic $2 \times 2$ square. Whenever the moving particle comes closer to one of the scatterers, with $r<1$ that scatterer exerts a smooth repulsive force:

$$
\begin{aligned}
& \phi(r<1)=\left(1-r^{2}\right)^{4} \longleftrightarrow F_{x}= \\
= & -8 x\left(1-r^{2}\right)^{3} ; F_{y}=-8 y\left(1-r^{2}\right)^{3}[r<1] .
\end{aligned}
$$

Although the 200,000 $(x, y)$ trajectory points appear to cover the space homogeneously at the left, a glancing initial condition is not ergodic and generates the 200,000-point torus shown at the right. At the right $p_{x}$ remains positive forever!

Ergodicity also implies that any initial condition must lead to the same time-averaged value of the largest Lyapunov exponent, $\lambda_{1}=\left\langle\lambda_{1}(t)\right\rangle$. This time-averaged exponent describes the rate at which the separation of two nearby phase-space trajectories tends to increase $[8,9]$ :

$$
\dot{\delta}=\lambda_{1}(t) \delta ; \delta \equiv \sqrt{\sum\left(\delta_{q}^{2}+\delta_{p}^{2}\right)} .
$$

\section{ERGODICITY VIA ISOENERGETIC ROTATIONS OF THE MOMENTA}

Although one might think, as we did, that continuous (but small) Coriolis accelerations,

$$
\left(\dot{p}_{x}, \dot{p}_{y}\right) \propto\left(+p_{y},-p_{x}\right)
$$

would lead to ergodicity, trials of this idea were unsuccessful. This failure led us to a successful and simpler conservative approach to ergodicity. We used discontinuous, rather than 

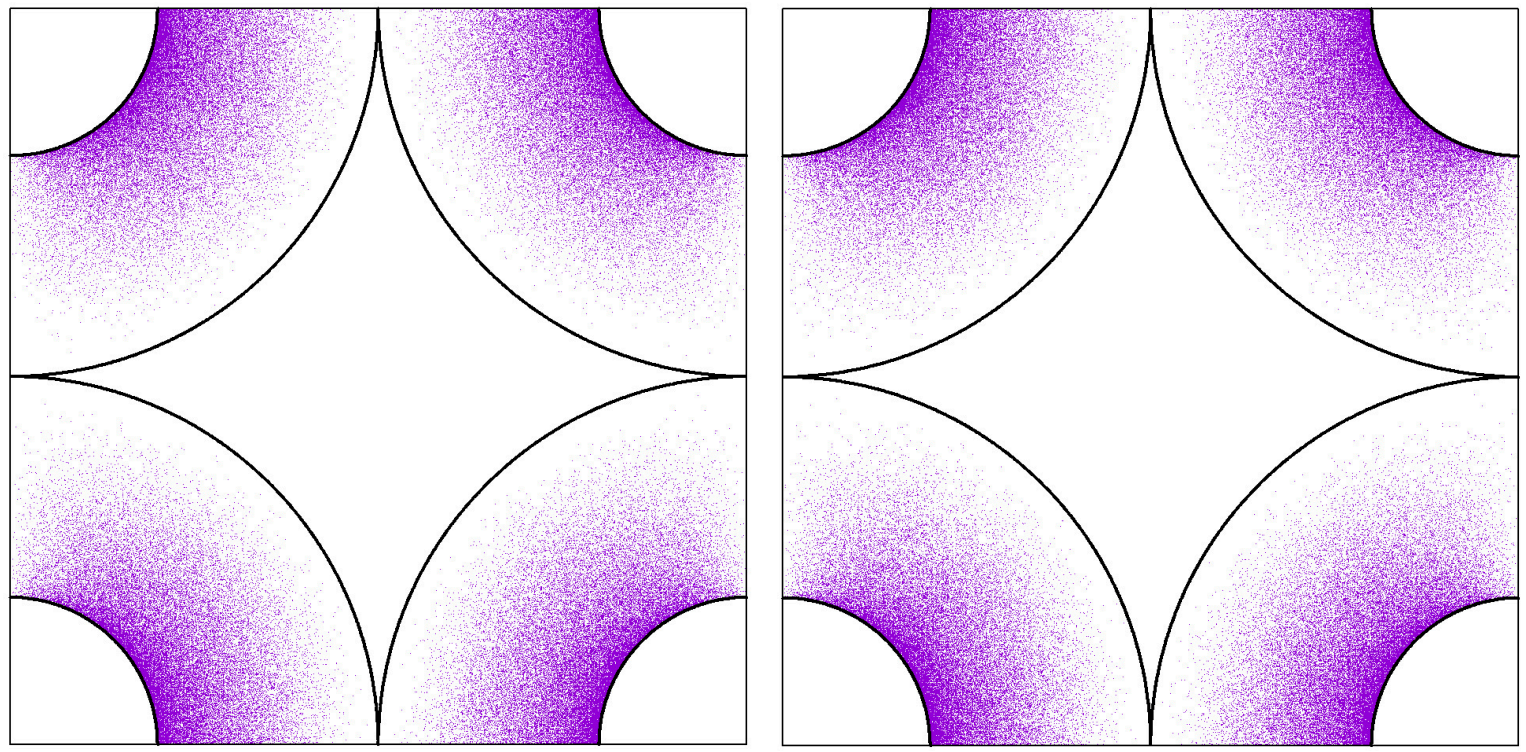

Fig. 2. The two Poincaré sections shown here are equally likely Cell-Model states with $p_{x}=0$. Unlike the uniform density of Fig. 1 there is no density at all in the central diamond, where the forces vanish, and the probability on the section is maximized along with the magnitude of the force at the scatterer boundaries where the velocity vanishes. Here the initial condition corresponds to the right panel of Fig. $1,\left(p_{x}, p_{y}\right)=\left(0.999, \sqrt{1-0.999^{2}}\right)$. The trajectory undergoes a random change of direction every ten steps at the left and every thousand at the right. The Sections shown, 182,749 points at the left and 183,342 points at the right correspond to one billion timesteps, $0<$ time $<1,000,000=1,000,000,000 d t$

smooth, random rotations of the moving particle's momentum. Let us indicate the change of momentum in two spatial dimensions:

$$
\left(\delta p_{x}, \delta p_{y}\right) \propto[\mathcal{R}-(1 / 2)] ; \mathcal{R}=\operatorname{rund}(\text { intx }, \text { inty })
$$

where the random numbers $\{0 \leq \mathcal{R}<1\}$ come from a simple time-reversible [10] generator:

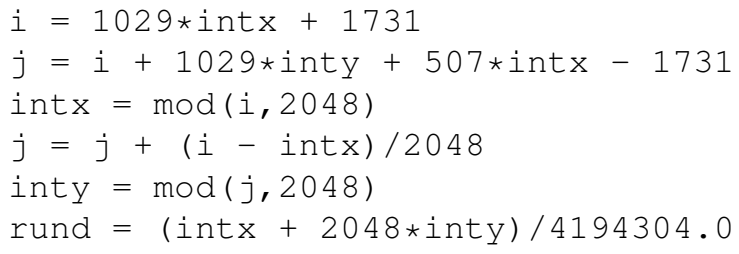

Because only the magnitude and not the orientation of a particle's momentum contributes to the energy such an algorithm is easily implemented. We have confirmed that even the smallest of systems can be made ergodic in this way, as indicated by the cell-model results in the following Section. Because the orientations of the single-particle momenta can be chosen or changed randomly we refer to the corresponding algorithm as a "Monte-Carlo" method, following the tradition of Metropolis, in his work with the Rosenbluths and Tellers [11].

\section{GENERATION OF MICROCANONICAL POINCARÉ SECTIONS}

Fig. 2 shows two $\left(p_{x}=0\right)$ sections obtained from MonteCarlo evaluations of the microcanonical phase-space density for a moving particle with $E=\Phi+K=(1 / 2)$. The cellmodel potential energy $\Phi$ describes the interaction of the moving or "wanderer" particle with four soft-disk scatterers at $\{ \pm 1, \pm 1\}$, the four vertices of a $2 \times 2$ square. The pair potential governing the scattering is $\phi=\left(1-r^{2}\right)^{4}$. Rather than show numerically that the entire microcanonical distribution is achieved in this way we will rely instead on the Metropolis, Rosenbluths, and Tellers proof of convergence of their canonical algorithm [11]:

"Since a particle is allowed to move to any point within a square of side 2 with a finite probability, it is clear that a large enough number of moves will enable it to reach any point in the complete square. Since this is true of all particles, we may reach any point in configuration space. Hence, the method is ergodic."

The simplicity of this algorithm recommends its use.

\section{References}

[1] S. Nosé, A Molecular Dynamics Method for Simulation in the Canonical Ensemble, Molecular Physics, 52, 255-268 (1984). 
[2] S. Nosé, A Unified Formulation of the Constant-Temperature Molecular Dynamics Methods, Journal of Chemical Physics, 81, 511-519 (1984).

[3] Wm.G. Hoover, Canonical Dynamics: Equilibrium PhaseSpace Distributions, Physical Review A, 31, 1695-1697 (1985).

[4] Wm.G. Hoover, B.L. Holian, Kinetic Moments Method for the Canonical Ensemble Distribution, Physics Letters A 211, 253257 (1996).

[5] G.J. Martyna, M.L. Klein, M. Tuckerman, Nosé-Hoover Chains: the Canonical Ensemble via Continuous Dynamics, The Journal of Chemical Physics 97, 2635-2643 (1992).

[6] Wm. G. Hoover, C.G. Hoover, J.C. Sprott, Nonequilibrium Systems: Hard Disks and Harmonic Oscillators Near and Far from Equilibrium, Molecular Simulation, 42, 1300-1316 (2016).

[7] D. Tapias, A. Bravetti, D.P. Sanders, Ergodicity of One-Dimensional Systems Coupled to the Logistic Thermostat, Computational Methods in Science and Technology 23, 11-18 (2017).
[8] I. Shimada, T. Nagashima, A Numerical Approach to Ergodic Problems of Dissipative Dynamical Systems, Progress of Theoretical Physics 61, 1605-1616 (1979).

[9] G. Benettin, L. Galgani, A. Giorgilli, J.-M. Strelcyn, Lyapunov Characteristic Exponents for Smooth Dynamics Systems and for Hamiltonian Systems; a Method for Computing All of Them, Parts I and II: Theory and Numerical Application, Meccanica 15, 9-20 and 21-30 (1980).

[10] F. Ricci-Tersenghi, The Solution to the Challenge in 'TimeReversible Random Number Generators' ar $\chi$ iv:1305.1805 (2013).

[11] N. Metropolis, A.W. Rosenbluth, M.N. Rosenbluth, A.H. Teller, E. Teller, Equation of State Calculations by Fast Computing Machines, The Journal of Chemical Physics 21, 1087-1092 (1953).

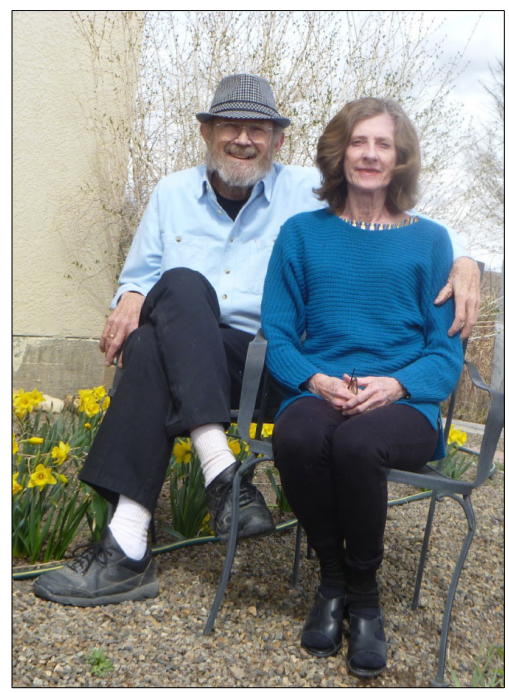

William G. Hoover, Carol G. Hoover; Bill and Carol Hoover live in Ruby Valley (Elko County Nevada) where the cows outnumber the people by orders of magnitude and the phonebook is a single page. Their research interests include chaos, fractals, symmetry breaking instabilities, and computer simulations linking the microscopic and macroscopic descriptions of nonequilibrium processes. 